AperTO - Archivio Istituzionale Open Access dell'Università di Torino

\title{
Acute kidney injury associated with glomerular diseases
}

\section{This is the author's manuscript}

Original Citation:

Availability:

This version is available http://hdl.handle.net/2318/1723438

since 2020-01-16T15:53:04Z

Published version:

DOI:10.1097/MCC.0000000000000675

Terms of use:

Open Access

Anyone can freely access the full text of works made available as "Open Access". Works made available under a Creative Commons license can be used according to the terms and conditions of said license. Use of all other works requires consent of the right holder (author or publisher) if not exempted from copyright protection by the applicable law. 


\section{IIIS AperTO}

UNIVERSITÀ

DEGLI STUDI

DI TORINO

This is the author's final version of the contribution published as:

Curr Opin Crit Care. 2019 Dec;25(6):573-579. doi: 10.1097/MCC.0000000000000675.

Acute kidney injury associated with glomerular diseases.

Fenoglio R, Sciascia S, Baldovino S, Roccatello D.

The publisher's version is available at:

https://insights.ovid.com/crossref?an=00075198-201912000-00007

When citing, please refer to the published version.

Link to this full text:

http://hdl. handle.net/2318/1723438

This full text was downloaded from iris-Aperto: https://iris.unito.it/ 


\section{AKI associated with glomerular diseases}

\section{Authors:}

Roberta Fenoglio, Savino Sciascia, Simone Baldovino, Dario Roccatello.

Nephrology and Dialysis Unit and CMID, Center of Research of Immunopathology and Rare Diseases Coordinating Center of the Network for Rare Diseases of Piedmont and Aosta Valley, Department of Clinical and Biological Sciences, University of Turin, San Giovanni Hospital and University of Turin, Turin, Italy

\section{Author of correspondence:}

\section{Dario Roccatello,}

Adress: Nephrology and Dialysis Unit and CMID, University of Turin and San Giovanni Hospital, Piazza del Donatore di Sangue 3, 10054, Turin, Italy

Telephone number: +390112402056

Email address: dario.roccatello@unito.it

\section{Abstract}

Purpose of review: the present review focuses on AKI associated with glomerular diseases and specifically the mechanisms of development of AKI in the wide spectrum of glomerulopathies.

Recent findings: the immune system and the kidneys are closely linked. In healthy subjects the kidneys contribute to immune homeostasis, while components of the immune system mediate many acute forms of kidney disease. Both crescentic and non-crescentic forms of acute glomerulonephritis (AGN) can present as AKI. The diagnosis of glomerular pathologies underlying AKI requires a high degree of 
suspicion coupled with an algorithmic approach to laboratory investigations. Renal biopsy represents the gold standard for the diagnosis of medical conditions of the kidney. The main clinical-biological presentations of glomerular diseases are acute nephritic syndrome, nephrotic syndrome and rapidly progressive glomerulonephritis. All these presentations can be worsened by AKI both in the onset and in the clinical course. Heavy proteinuria and macroscopic hematuria can be directly involved in the development of AKI.

Summary: AKI associated with glomerular diseases is not uncommon. Sometimes it represents an emergency case. The understanding of the various mechanisms underlying kidney diseases is improving, and may aid in their prevention and treatment

\section{Key words:}

AKI, red blood cell casts, nephrotic syndrome, rapidly progressive glomerulonephritis.

\section{Abbreviations:}

Acute kidney injury (AKI), glomerular filtration rate (GFR), acute glomerulonephritis (AGN), nephrotic syndrome (NS), rapidly progressive glomerulonephritis (RPGN), IgA nephropathy (IgAN), glomerulonephritis (GN), minimal change disease (MCD), C3 glomerulonephritis (C3GN), hemoglobin (Hb), MicroRNA (miRNA), acute tubular necrosis (ATN), anti-glomerular basement membrane disease (anti-GBM disease), ANCA-associated vasculitis (AAV), neutrophil extracellular traps (NETs), complement alternative pathway (cAP) 


\section{Introduction}

The classification of acute kidney injury (AKI) includes pre-renal AKI, acute post-renal obstructive nephropathy and intrinsic acute kidney diseases [1]. Of these, only intrinsic AKI represents true kidney disease, while pre-renal and post-renal AKI are the consequence of extra-renal pathological conditions leading to decreased glomerular filtration rate (GFR). If these pre-and/or post-renal conditions persist, they will eventually evolve to cellular damage, and hence intrinsic kidney disease. Intrinsic kidney etiologies of AKI can be challenging to evaluate because of the wide variety of injuries that can occur to the kidney. Tubules, glomeruli, interstitium, and intra-renal blood vessels are the four structures of the kidney generally involved. The glomerulopathies account for $10 \%$ of AKI in adults [2].

The spectrum of glomerulopathies presenting as AKI varies depending on many factors, such as age, sex, race and geographical area, as well as biopsy policies [2,3].

The immune system and the kidneys are closely linked. In healthy subjects the kidneys contribute to immune homeostasis, while components of the immune system mediate many acute forms of kidney disease. Both crescentic and non-crescentic forms of acute glomerulonephritis (AGN) can present as AKI. The diagnosis of glomerular pathologies underlying AKI requires a high degree of suspicion coupled with an algorithmic approach to laboratory investigations. Kidney biopsy currently represents the gold standard for the diagnosis of medical conditions of the kidney.

AKI can be mediated both by pathogenic mechanisms and by clinical signs of damage such as proteinuria and hematuria. The main clinical-biological presentations of glomerular diseases are acute nephritic syndrome, nephrotic syndrome (NS) and rapidly progressive glomerulonephritis (RPGN) [Table I].

\section{Hematuria and AKI}

Gross hematuria promotes impairment of kidney function by different pathological mechanisms [4]. IgA 
nephropathy (IgAN), the commonest primary glomerulonephritis (GN), is the most frequent cause of glomerular hematuria [5]. Approximately half of patients may present with gross hematuria, while the other half may present with microhematuria. Rapidly progressive glomerulonephritis, small size vessel vasculitis, and acute glomerular inflammation, as observed in post-infectious GN or lupus, may also be associated with glomerular hematuria. Hematuria is frequent in endocapillary and extracapillary GN, but the real prevalence is mainly based on observational cohorts [6,7]. Data from the Spanish renal biopsy registry [5] reported an unexpectedly high rate of hematuria (50\%) among patients who were traditionally considered not to have hematuria as a presentation sign, including minimal change disease (MCD), membranous GN, or focal and segmental glomerulosclerosis, but the occurrence of gross hematuria is extremely unfrequent. In the newly characterized C3 glomerulonephritis (C3GN), hematuria-mainly microscopic —was also present in a high percentage of patients [8]. AKI can be due to massive hematuria of glomerular origin. The damage to the tubular cells is caused by both the intratubular obstruction of the blood casts and by processes of erythrophagocytosis by the tubular cells. Thus, the duration of macrohematuria becomes a crucial phenomenon for the recovery of renal function [9]. Red blood cells release hemoglobin ( $\mathrm{Hb}$ ) products, which are further taken up by tubular cells. Once inside the cell, $\mathrm{Hb}$ dissociates, releasing the globins and the heme group, thereby inducing several pathological effects, including oxidative stress, cell death, inflammation, and fibrosis [4]. Recently, RubioNavarro et al showed that in addition to the tubular cells, podocytes may be the cellular target of $\mathrm{Hb}$ mediated kidney damage. Thus, $\mathrm{Hb}$ induces oxidative damage, podocyte dysfunction, and finally apoptosis, and the detachment of the podocyte from the glomerular capillary [10]. The traffic of $\mathrm{Hb}$ through the capillary wall can damage the podocyte, and may result in an alteration of the glomerular filtration barrier. Erythrocyte injury following distortion through the leaks of the glomerular filtration barrier promotes the release of microvesicles containing microRNA (miRNA). miRNA can be swallowed by nearly all cells, and plays an important role in the regulation of oxidative stress and intercellular 
communication by regulating gene expression. The more prevalent miRNA detected in the urine of IgAN patients with hematuria was mainly derived from urinary erythrocytes. Hematuria-associated miRNA could change gene expression of parenchymal cells and promote the evolution of kidney disease [11]. The role of miRNA in the pathogenesis of hematuria-associated AKI remains speculative and it will need to be confirmed by further studies.

\section{Nephrotic syndrome and AKI}

Recent years have seen an increase in the incidence of AKI in NS. AKI is more commonly reported in adults with idiopathic NS but it can potentially complicate all cases of nephrotic syndrome regardless of the nephropathy that causes it [12].

AKI in NS is generally reversible and its pathogenesis is probably multifactorial. Several mechanisms have been proposed over the year. Poorly identified factors that reversibly affect the glomerular capillary framework in patients with NS, especially those with minimal change disease, lead to an increased permeability of the glomerular basement membrane to serum albumin and reduce its permeability to water and small molecules [13]. Until recent years most information on the epidemiology of AKI in NS came from case reports. By examining 79 cases of AKI in 75 patients with minimal change disease and a mean age of 58 years reported in the literature since 1966, Smith et al [14] showed that the average time for occurrence of AKI was $29 \pm 5$ days after the onset of nephrotic syndrome. In the 58 patients who recovered renal function, AKI lasted for 7 weeks. While AKI was attributed to volume depletion, most patients did not improve after correcting volume depletion, and $60 \%$ of cases showed histopathological changes consistent with acute tubular necrosis (ATN). Conversely, in a more recent study on idiopathic nephrotic syndrome, recovery of renal function occurred in the vast majority of 277 patients [12]. More severe AKI was associated with longer time to remission and lower rates of complete remission. Severe hypoalbuminemia, older age, and male gender were identified as risk factors on logistic regression 
analysis. A more intense endothelin 1 immunoreactivity was detected in vessels, tubules, and glomeruli in AKI compared to non-AKI group. Endothelin 1 acts on the kidney vasculature, inducing a decrease in blood flow and GFR. Patients with NS and AKI could have suffered of transient episodes of circulatory insufficiency at the onset of NS. On the other hand, tubular cells could have reabsorbed filtered proteins. Consonant with this hypothesis is the observation that in the most severe cases of AKI a process of kidney ischemia is operating especially in elderly and/or hypertensive patients with nephrosclerosis (an "umbrella term" covering several microvascular lesions) that reduce the afferent arteriolar blood supply to the glomerular tuft. These microvascular lesions along with inflammatory interstitial fibrosis and tubular atrophy compromise tubule viability [15]. Thus the traditional underfill hypothesis with reduction of the effective plasma flow is no longer believed to be responsible for reduced GFR.

A pathophysiologic mechanism that has been assumed as a dogma over several years ascribed sodium retention and renal insufficiency in nephrotic syndrome to a state of hypovolemia. Actually, in steadystate conditions, i.e., in the absence of excessive loop-diuretic treatment, blood volume is not reduced. In nephrotic edema a new equilibrium is achieved between a lower plasma albumin concentration and higher pressure in the interstitial fluid. In this setting interstitial fluid is taken up by the lymphatic system and the increased lymph flow maintains plasma volume close to normal [16]. Other postulated pathogenic mechanisms for AKI in NS have been no longer supported. Some clinical observations supported the hypothesis that high urine hyperviscosity resulting from very high albumin concentrations may be $\underline{a}$ cause of oliguria [17]. The so called nephrosarca theory suggested that interstitial edema in NS induced tubular collapse. Ischemic tubular injury and tubular necrosis has been reported in certain series but the underlying mechanism remains unclear [18]. Recently Fujigaki et al. examined the changes of tubular cells in 37 adult patients with MCD [19]. Thirteen out of 37 (35.1\%) had AKI and were found to have a significant increase in some tubular injury markers (such as urinary $\mathrm{N}$-acetyl- $\beta$-D-glucosaminidase and 
urinary alpha1-microglobulin). The immunohistochemical expression of vimentin, another marker of tubular injury, was found to be significantly increased in the AKI group.

\section{Rapid progressive glomerulonephritis and AKI}

RPGN is an emergency setting. Early diagnosis and treatment improve prognosis, and, at the beginning of therapy, creatinine is the best prognostic marker [20]. Classical examples of immune-related diseases which present with RPGN are anti-glomerular basement membrane disease (anti-GBM disease) and, ANCA-associated vasculitis (AAV). Anti-GBM disease is a form of crescentic glomerulonephritis presenting with acute kidney abnormalities, and often with severe alveolar hemorrhage (Goodpasture's syndrome) . Circulating autoantibodies bind the NC1 domain of the collagen IV a3 chain. Potential triggers for the unmasking of the autoantigen are still unknown, but once binding has occurred it leads to further conformational changes perpetuating the antigen-antibody complex formation. The resulting linear immune deposits along the glomerular basement membranes, typically composed of IgG and complement components, damage the surrounding endothelial cells and podocytes leading to immune cell infiltration [21].

AAV with kidney involvement present with hematuria, proteinuria and rapid impairment of filtration function, and is characterized by damage of the glomerular vasculature. AAV runs a rapidly progressive course which consonant with a crescentic glomerular morphology on kidney biopsy with severe necrotizing destruction of the glomerular tufts [22].

The development of the pathological lesions in AAV is uncompletely defined. It may be initiated by glomerular injury, which activates neutrophils leading to degranulation and expulsion of neutrophil extracellular traps (NETs) as the first step of the tissue repair process. The extrusion of NETs into the glomerular capillaries promotes the release the ANCA-associated antigens (including myeloperoxidase, 
proteinase 3 and lysosome-associated membrane glycoprotein 2), which are subsequently bound by circulating ANCA antibodies [23]. Furthermore, in vivo studies have shown that PR3-ANCA can activate circulating neutrophils directly. Autoreactive T cells have also been implicated as important factors in the pathogenesis of AAV [24]. Reactive oxygen species (ROS) released by activated neutrophils, autoantibody binding to NETs and extensive activation of leukocytes cause local inflammation and complement activation within glomerular capillaries. This vascular inflammation leads to focal necrosis with destruction of the glomerular barrier, clinical appearance of hematuria and rapidly diminishing renal function $[25,26]$.

Macrophages are myeloid immune cells that are positioned throughout the body tissues and derive from circulating monocytes. Upon activation, macrophages can turn either into classically activated (M1) macrophages, which promote tissue injury, or alternatively activated (M2) macrophages, which promote tissue repair [27]. Zhao et al [28], showed CD68+ and CD163+ macrophagesto be predominant at sites of fibrinoid necrosis, exceeding the quantity of neutrophils and $\mathrm{T}$ cells. Furthermore, in AAV patients normal-appearing glomeruli had significantly more CD68+ and CD163+ macrophages than controls. The Authors hypothesized that M2 macrophages might be precursor steps in the evolution of fibrinoid necrosis and subsequent crescent formation. Rousselle et al [29] found that monocytes were able to promote crescent formation in a mouse model of AAV. These studies clearly suggest macrophages/monocytes to be related to vasculitic kidney damage.

Complement deposition was found to be associated with cellular crescents as well $[30,31]$ and could play a role in AAV. During the development of segmental fibrinoid necrosis, interstitial monocytes and CD3+ T cells infiltrated and then contributed to further kidney damage. The role of the complement system has recently regained importance in the pathogenesis of kidney diseases. Atypical hemolytic uremic syndrome and some forms of membranoproliferative glomerulonephritis are clearly secondary to genetic 
abnormalities or circulating antibodies that impair the activity of the redundant regulatory proteins of the complement alternative pathway (cAP) (e.g., complement Factor H, complement Factor B, MCP) [32]. Moreover, other forms of glomerulonephritis (e.g., lupus nephritis, cryoglobulinemic glomerulonephritis)[33] are triggered by complement activation. Recently, AAV was recently added to this list [34]. Studies performed in the past few years showed that most patients have at least focal complement deposition at the sites of injury, and that complement glomerular deposition (particularly C3) is associated with poorer renal function and prognosis. These findings were elicited in vivo studies that used murine models of AAV, and showed activation of the terminal part of the complement cascade, particularly of C5. The pathogenic relevance of the complement system in ANCA-associated necrotizing crescentic glomerulonephritis is confirmed by immunohistochemical studies that demonstrate the presence of glomerular and vascular immune deposits consisting of complement fragments. A dominant role of the cAP in AAV is hypothesized based on the higher plasma levels of $\mathrm{C} 3 \mathrm{a}$ and $\mathrm{Bb}$ in active AAV cases than in quiescent ones [Table II].

\section{Other mechanisms of AKI associated with glomerular diseases}

The majority of immune-mediated kidney pathologies are due to mechanisms of dysregulated autoimmunity (Fig.1), but some are the consequence of genetic defects in the complement cascade or hematological malignancies, such as multiple myeloma or Hodgkin lymphoma. Kidney injury secondary to loss of immune homeostasis is caused by three major mechanisms: circulating immune complex deposition, dysregulation of the alternate complement pathway and deposition of monoclonal immunoglobulins, although others are implicated, such as local elevation of cytokine concentration in podocytopathies and autoantibodies targeting to systemic vasculature causing downstream ischemic damage in thrombotic microangiopathies.

Immune complexes damage the glomerular endothelium, epithelium and mesangium by activating 
circulating immune cells and kidney-intrinsic cells expressing Fc receptors. Activated cells secrete cytokines and vasoactive substances to create a proinflammatory environment following immune complex deposition. In addition, the complement cascade is activated via the classical pathway, the endpoint of the cascade being the formation of the membrane attack complex, which injures surrounding cells, leading to further proinflammatory signaling. Examples of glomerulonephritis caused by immunocomplex deposition are IgA nephropathy, Lupus nephritis, membranoproliferative glomerulonephritis or infection-related glomerulonephritis [23].

Over-activity of the complement system leading to kidney damage can be due to genetic mutations in complement regulatory components or autoimmune disease against components of the complement system [35].

C3 deposits can form in the glomerulus (Fig.1), activating downstream inflammatory cascades and promoting leukocyte infiltration. In dense deposit disease, small bandlike electron dense deposits of complement form along the GBM, leading to GBM thickening and dysfunction. In addition to GBM deposits, C3 glomerulopathies also show varying degrees of mesangial C3 deposits, leading to mesangial cell proliferation and matrix expansion. An example of glomerulonephritis caused by C3 deposition is C3 glomerulopathies [36][Table II].

Injury of the kidney vascular tree can also be mediated by conditions related to the loss of immune homeostasis. Hemolytic uremic syndrome (HUS), either the classical diarrhoea-associated type or atypical non-diarrhoea-associated HUS can manifest with AKI. Similar-appearing HUS lesions can be caused by thrombotic microangiopathies. Primary anti-phospholipid syndrome (APS) is an autoimmune disease characterized by circulating anti-phospholipid antibodies (aPLs), recurrent venous or arterial thrombosis and pregnancy-related problems. aPLs, which include lupus anti-coagulant (LA), anticardiolipin (aCL) and antib2-glycoprotein I (anti-b2GPI), create a procoagulant state, which leads to 
thrombosis in the presence of a further prothrombotic factor, such as oxidative stress, surgical intervention or infection. Approximately $10 \%$ of patients with primary APS develop kidney involvement, presenting in a small percentage of cases with AKI [37].

Finally, AKI can occur in patients with mixed cryoglobulinemia and nephritis as a consequence of microvascular obliteration by cryoglobulins [38].

\section{Conclusion}

AKI associated with glomerular diseases is not uncommon. Sometimes it represents an emergency case. The understanding of the various mechanisms underlying kidney diseases is increasing, and may aid in their prevention and treatment.

\section{Key points:}

1. The classification of acute kidney injury (AKI) includes pre-renal AKI, acute post-renal obstructive nephropathy and intrinsic acute kidney diseases (1). Of these, only intrinsic AKI represents true kidney disease, while pre-renal and post-renal AKI are the consequence of extra-renal pathological conditions leading to decreased glomerular filtration rate (GFR).

2. The diagnosis of glomerular pathologies underlying AKI requires a high degree of suspicion coupled with an algorithmic approach to laboratory investigations. Kidney biopsy currently represents the gold standard for the diagnosis of medical conditions of the kidney.

3. Gross hematuria and nephrotic syndrome promote impairment of renal function by different pathological mechanisms.

4. RPGN is an emergency setting. Early diagnosis and treatment improve prognosis, and, at the beginning of therapy, creatinine is the best prognostic marker (19). Classical examples of immune- 
related diseases which present with RPGN are anti-glomerular basement membrane disease (antiGBM disease) and, ANCA-associated vasculitis (AAV)

5. The majority of immune-mediated kidney pathologies are due to mechanisms of dysregulated autoimmunity, but some are the consequence of genetic defects in the complement cascade or hematological malignancies, such as multiple myeloma or Hodgkin lymphoma.

\section{Acknowledgements:}

None

Financial support and sponsorship:

None

\section{Conflicts of interest:}

None 


\section{REFERENCES}

1. Makris K, Spanou L. Acute Kidney Injury: Definition, Pathophysiology and Clinical Phenotypes. Clin Biochem Rev. 2016; 37(2): 85-98.

2. Thervet É. Acute renal failure of glomerular origin. Rev Prat. 2018; 68(2):160-165.

3. Roccatello D, Sciascia S, Rossi D, et al. Safety of outpatient percutaneous native renal biopsy in systemic autoimmune diseases: results from a monocentric cohort. Lupus. 2018; 27(8):1393-1394.

This subsequent analysis provides evidence that the biopsy in patients with renal diseases is mandatory. A careful screening for bleeding risk, the use of an automated needle-gun system under ultrasound guide, and the presence of a standardized protocol minimize the risk for complications even as an outpatient procedure.

4. Moreno JA, Sevillano Á, Gutiérrez E, et al. Glomerular Hematuria: Cause or Consequence of Renal Inflammation? Int J Mol Sci. 2019; 5;20(9). pii: E2205. doi: 10.3390/ijms20092205

5. Yuste C, Rivera F.; Moreno JA et al. Haematuria on the Spanish Registry of Glomerulonephritis. Sci. Rep. 2016; 6: 19732.

6. Wyatt RJ, Julian BA. IgA Nephropathy. N. Engl. J. Med. 2013; 368: 2402-2414.

7. Sethi S, Zand L, Nasr SH, et al. Focal and segmental glomerulosclerosis: Clinical and kidney biopsy correlations. Clin. Kidney J. 2014; 7, 531-537.

8. Medjeral-Thomas NR, O'Shaughnessy MM, O'Regan JA, et al. C3 glomerulopathy: Clinicopathologic features and predictors of outcome. Clin. J. Am. Soc. Nephrol. 2014; 9: 46-53. 
9. Schulman G., Berl T., Beck G.J., et al. Risk factors for progression of chronic kidney disease in the EPPIC trials and the ect of AST-120. Clin. Exp. Nephrol. 2018; 22: 299-308.

10. Rubio-Navarro A., Sanchez-Niño M.D., Guerrero-Hue, M., et al. Podocytes are new cellular targets of haemoglobin-mediated renal damage. J. Pathol. 2018; 244.

11. Duan Z.Y., Cai G.-Y, Bu, R, et al. Selection of urinary sediment miRNAs as specific biomarkers of IgA nephropathy. Sci. Rep. 2016; 6: 23498. doi: 10.1038/srep2349.

12. Chen T, Lv Y, Lin F, et al. Acute kidney injury in adult idiopathic nephrotic syndrome. Renal Fail. 2011; 33:144-9.

13. Maas RJ, Deegens JK, Wetzels JF. Permeability factors in idiopathic nephrotic syndrome: historical perspectives and lessons for the future. Nephrol Dial Transplant. 2014; 29:2207-2216.

14. Smith JD, Hayslett JP. Reversible renal failure in the nephrotic syndrome. Am J Kidney Dis. 1992; 19:201-13.

15. Meyrier A. Nephrosclerosis: a term in quest of a disease. Nephron. 2015; 129:276-282.

16. ${ }^{\circ}$ Meyrier A, Niaudet P. Acute kidney injury complicating nephrotic syndrome of minimal change disease. Kidney Int. 2018; 94(5):861-869.

A complete review on possible pathogenetic mechanisms of AKI associated with nephrotic syndrome

17. Koomans HA. Pathophysiology of acute renal failure in idiopathic nephrotic syndrome. Nephrol Dial Transplant. 2001; 16:221-224.

18. Koomans HA. Pathophysiology of acute renal failure in idiopatic nephrotic syndrome. Nephrol Dial Transplant. 2001; 16:221-4. 
19. Fujigaki $Y$, Tamura $Y$, Nagura $M$, et al. Unique proximal tubular cell injury and the development of acute kidney injury in adult patients with minimal change nephrotic syndrome. BMC Nephrol. 2017; 18:339.

20. Haubitz M. Rapid progressive glomerulonephritis. Internist (Berl). 2019; 60(5):478-484.

21. ${ }^{\circ}$ Tecklenborg J, Clayton D, Siebert S, et al The role of the immune system in kidney disease Clinical and Experimental Immunology 2018; 192: 142-150.

An important overview on the close correlations between the immune system and the kidney.

22. Alba MA, Jennette JC, Falk RJ. Pathogenesis of ANCA-Associated Pulmonary Vasculitis. Semin Respir Crit Care Med. 2018; 39(4):413-424.

23. Kurts C, Panzer U, Anders HJ, et al. The immune system and kidney disease: basic concepts and clinical implications. Nat Rev Immunol 2013; 13:738-53.

24. Kerstein A, Muller A, Kabelitz D, et al. Effector memory T-cells in the pathogenesis of ANCA-associated vasculitis. Rheumatol 2017; 76:14.

25. Chen Y.X., Zhang W., Chen X.N. Application of RIFLE criteria in Chinese patients with ANCA-associated renal vasculitis. Clin Exp Rheumatol. 2011; 29:951-957.

26. Chen YX, Chen XN. Antineutrophil cytoplasmic antibodies-associated glomerulonephritis: From bench to bedside. Chronic Dis Transl Med 2018; 16; 4(3):187-191.

27. Varol C, Mildner A, Jung S. Macrophages: development and tissue specialization. Annu Rev mmunol. 2015; 33:643-675.

28. Zhao L, David M.Z, Hyjek E, et al. M2 macrophage infiltrates in the early stages of ANCAassociated pauci-immune necrotizing GN. Clin J Am Soc Nephrol. 2015; 10:54-62. 
29. Rousselle A., Kettritz R., Schreiber A. Monocytes promote crescent formation in antimyeloperoxidase antibody-induced glomerulonephritis. Am J Pathol. 2017; 187:19081915.

30. Hilhorst M, van Paassen $P$, van Rie H. Complement in ANCA-associated glomerulonephritis. Nephrol Dial Transplant. 2017; 32:1302-1313.

31. Chen M, Xing GQ, Yu F, et al. Complement deposition in renal histopathology of patients with ANCA-associated pauci-immune glomerulonephritis. Nephrol Dial transplant. 2009; $24: 1247-1252$.

32. Angioi A, Fervenza F, Sethi S, et al. Diagnosis of complement alternative pathway disorders. Kidney Int 2016; 89(2):278-288.

33. ${ }^{\circ}$ Rovin BH, Caster DJ, Cattran DC, et al. Management and treatment of glomerular diseases (part 2): conclusions from a Kidney Disease: Improving Global Outcomes (KDIGO) Controversies Conference. Kidney Int. 2019; 95(2):281-295.

A recent update of the $2012 \mathrm{KDIGO}$ glomerulonephritis guideline in the context of new developments and insights that had occurred over the years since its publication. This report summarizes the discussions about pathogenesis, biomarkers, and treatments of primary podocytopathies, lupus nephritis, anti-neutrophil cytoplasmic antibodyassociated nephritis, complement-mediated kidney diseases, and monoclonal gammopathies of renal significance.

34. Manenti L, Vaglio A, Gnappi E, et al . Association of serum C3 concentration and histologic signs of thrombotic microangiopathy with outcomes among patients with ANCAassociated renal vasculitis. Clin J Am Soc Nephrol 2015; 10(12):2143-2151. 
35. Lionaki S, Gakiopoulou H, Boletis JN. Understanding the complement-mediated glomerular diseases: focus on membranoproliferative glomerulonephritis and C3 glomerulopathies. APMIS 2016; 124:725-35.

36. ${ }^{\circ}$ Smith RJH, Appel GB, Blom AM, et al. C3 glomerulopathy understanding a rare complement-driven renal disease. Nat Rev Nephrol. 2019; 15(3):129-143.

An update review on classification, pathogenesis, clinical presentation, and management of C3 glomerulopathy

37. ${ }^{\circ}$ Schreiber K, Sciascia S, de Groot PG, et al. Antiphospholipid syndrome. Nat Rev Dis Primers. 2018; 4:18005. doi: 10.1038/nrdp.2018.5

A complete review on definition, pathogenesis, clinical presentation, treatment with conventional and innovative treatment of Antiphospholipid syndrome.

38. ${ }^{\circ}$ Roccatello D, Saadoun D, Ramos-Casals M, et al. Cryoglobulinaemia. Nat Rev Dis Primers. 2018; 4(1):11. doi: 10.1038/s41572-018-0009-4.

A complete review on definition, pathogenesis, clinical presentation, treatment with DAAs and conventional and innovative treatment, and future perspective of cryoglobulinemia.

Fig. 1. Mechanisms of immune-related AKI. A) immune-complex deposits (mesangial, subendothelial, subepithelial), B) C3 deposition, C) Immunoglobulin deposition, D) cellmediated immune damage. Non immune-related mechanisms are described in Table I. 
